\title{
Alterstice
}

Revue internationale de la recherche interculturelle

International Journal of Intercultural Research

Revista International de la Investigacion Intercultural

\section{Alterstice souffle sa première bougie}

\section{Yvan Leanza}

Volume 2, numéro 2, 2012

URI : https://id.erudit.org/iderudit/1077559ar

DOI : https://doi.org/10.7202/1077559ar

Aller au sommaire du numéro

Éditeur(s)

Alterstice

ISSN

1923-919X (numérique)

Découvrir la revue

Citer ce document

Leanza, Y. (2012). Alterstice souffle sa première bougie. Alterstice, 2(2), 1-2.

https://doi.org/10.7202/1077559ar d'utilisation que vous pouvez consulter en ligne.

https://apropos.erudit.org/fr/usagers/politique-dutilisation/ 


\section{ज \\ alterstice}

Revue Internationale

de la Recherche Interculturelle

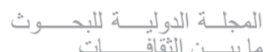

Revista International

de la Investigacion Intercultural

Revista Internacional

de Pesquisa Intercultural

International Journal

of Intercultural Research

ÉDITORIAL

\section{Alterstice souffle sa première bougie}

Yvan Leanza $^{1}$

Les premiers articles publiés par Alterstice étaient mis en ligne le 15 décembre 2011. Bien que le site et le système soient en opération depuis août 2010, cela fait à peine plus d'un an que les textes sont accessibles. Au cours de cette année d'existence virtuelle, quatre numéros ont été mis en ligne, deux pour l'année 2011 et deux pour l'année 2012. Cela représente 55 textes, dont 29 articles thématiques, 10 notes de lectures et 3 articles hors thème, mais aussi 4 introductions, 4 éditoriaux et 4 numéros complets, plus 1 entrevue croisée. Ces chiffres n'incluent que les textes acceptés pour publication. II y a bien entendu aussi des textes soumis et refusés suite à l'évaluation par le comité de direction ou les pairs. Ils sont au nombre de 25 à cette date, soit presque autant que d'articles acceptés et publiés.

Les articles publiés sont-ils lus? Telle est la question... Grâce au fait qu'Alterstice est une revue complètement virtuelle, il est possible d'avoir une idée de la fréquentation du site et surtout du nombre de téléchargements des articles. Les auteurs ont d'ailleurs dans leur session personnelle un compteur qui leur permet de savoir combien de fois le résumé a été consulté et l'article téléchargé. Évidemment ces chiffres ne disent rien de la vie des articles: s'ils sont utilisés dans l'enseignement, dans la formation, s'ils sont cités... Je compte sur les lecteurs et les auteurs pour me tenir informé de cet usage. Mais quel est donc le score d'Alterstice? En moyenne, chaque texte sur le site, quelle que soit sa catégorie (article thématique, introduction, etc.) est téléchargé une fois par jour, soit 365 fois par année. Les notes de lecture sont les textes les plus téléchargés (une moyenne d'un peu moins de 1,5 fois par jour), les éditoriaux les moins téléchargés (en moyenne une fois tous les deux jours), mais je ne m’en offusque point...

II me semble que c'est un score très honorable pour une revue naissante, dont le réseau de diffusion est constitué, en plus des 250 " abonnés », de quelques centaines de chercheurs - surtout francophones - participants à l'ARIC, au CELAT et à METISS, les trois institutions soutenant la revue. Étant donné le nombre de téléchargements, cela signifie que la diffusion se fait au-delà de ce réseau primaire. Un des objectifs de la revue, dans la lignée de la philosophie de I'ARIC, est de donner la possibilité aux chercheurs des pays du Sud d'avoir accès aux textes, mais aussi de proposer leurs travaux. Au vu des inscriptions sur le site, dont une part importante provient du Maghreb et de l'Amérique du Sud, cette politique atteint progressivement son but.

Tout ceci ne serait pas possible sans l'implication des éditrices et éditeurs invités, des évaluateurs et d'Anna Olivier, réviseure professionnelle, dont la disponibilité et le travail minutieux façonnent l'image d'Alterstice. La liste qui suit comprend toutes les personnes qui ont été invitées à évaluer un ou plusieurs articles et qui l’ont fait bénévolement. Qu'ils en soient ici chaleureusement remerciés. 
Abdeljalil Akkari; Pierre Anctil; Mathilde Anquetil; Nathalie Auger; Catherine Berger; Patrick Berteaux; Gilles Bibeau; Denis Blot; Brahim Boudarbat; Nawal Boudechiche; Camille Brisset; Claudine Brohy; Marie-Anne Broyon; Nicole Carignan; Line Chamberland; Nilima Changkakoti; Michèle Clément; Patrick Cloos; Marguerite Cognet; Pierre Dasen; Olivier Ducharme; Anahy Gajardo; Aline Gohard; Audrey Gonin; Myriam Gremion; Lucille Guilbert; Ghayda Hassan; Mondher Kilani; Marie-Christine Kok-Escalle; Ève Lamoureux; Marguerite Lavallée; Anne Lavanchy; Mouhamed Abdallah Ly; Marc Maesschalck; Altay Manço; Lucienne Martins Borges; Abdelwahed Mekki-Berrada; René Mokounkolo; Catherine Montgomery; Donatille Mujawamariya; Tania Ogay; Laurence Ossipow; Christiane Perregaux; Guylaine Racine; Jacques Rhéaume; Ilario Rossi; Andrew G. Ryder; Colette Sabatier; Francine Saillant; Soumya Tamouro; Fabienne Tanon; Pascal Tisserant; Bertrand Troadec; Josianne Veillette; Richard Walling; Patchareerat Yanaprasart; Jamil Zugueib.

Aujourd'hui, l'édition en ligne de revues savantes est un véritable commerce. Le modèle dominant veut que le lecteur paie pour avoir accès aux réflexions et résultats de recherche, ou que l'auteur paie pour rendre son travail de recherche accessible. Dans ce vaste marché, Alterstice a fait le choix de l'accès totalement gratuit. Ni les lecteurs, ni les auteurs n'ont à débourser le moindre kopeck pour que la revue vive. Ceci est possible grâce au soutien de I'ARIC, du CELAT et de METISS, et des institutions publiques de financement de la recherche qui ellesmêmes soutiennent ces réseaux. C'est une dimension importante de la philosophie de la revue. Pourvu que cela dure...

Ce numéro fait justement la part belle à l'équipe METISS, qui expose ici ses derniers travaux. Sous la direction de Catherine Montgomery et Soumya Tamouro, les chercheurs y présentent leurs observations et réflexions sur les diversités dans le vaste univers de la santé.

Bonne lecture!

\section{Rattachement de l'auteur}

${ }^{1}$ Université Laval, Québec, Canada

\section{Correspondance}

alterstice@gmail.com

\section{Pour citer cet article :}

Leanza, Y. (2012). Alterstice souffle sa première bougie [Éditorial]. Alterstice, 2(2), 1-2. 